\title{
Rehabilitation for a child with recalcitrant anti-N-methyl-D-aspartate receptor encephalitis: case report and literature review
}

This article was published in the following Dove Press journal:

Neuropsychiatric Disease and Treatment

24 November 2014

Number of times this article has been viewed

\author{
Yao-Hong Guo' \\ Ta-Shen Kuan ${ }^{1,2}$ \\ Pei-Chun Hsieh' \\ Wei-Chih Lien' \\ Chun-Kai Chang' \\ Yu-Ching Lin ${ }^{1-3}$ \\ 'Department of Physical Medicine \\ and Rehabilitation, National Cheng \\ Kung University Hospital, College \\ of Medicine, National Cheng \\ Kung University, Tainan, Taiwan; \\ ${ }^{2}$ Department of Physical Medicine \\ and Rehabilitation, College of \\ Medicine, National Cheng Kung \\ University, Tainan, Taiwan; ${ }^{3}$ Medical \\ Device Innovation Center, National \\ Cheng Kung University, Tainan, Taiwan
}

\begin{abstract}
Anti- $N$-methyl-D-aspartate (anti-NMDA) receptor encephalitis is a newly recognized, potentially fatal, but treatable autoimmune disease. Good outcome predictors include milder severity of symptoms, no need for intensive care unit admission, early aggressive immunotherapy, and prompt tumor removal. We report a case of a young girl aged 3 years 2 months and diagnosed as recalcitrant anti-NMDA receptor encephalitis without any underlying neoplasm. The patient had initial symptoms of behavioral changes that progressed to generalized choreoathetosis and orofacial dyskinesia, which resulted in 6 months of hospitalization in the pediatric intensive care unit. One year after initial onset of the disease, she had only achieved the developmental age of an infant aged 6-8 months in terms of gross and fine motor skills, but she resumed total independence in activities of daily living after receiving extensive immunotherapy and 28 months of rehabilitation. Our brief review will help clinical practitioners become more familiar with this disease and the unique rehabilitation programs.
\end{abstract}

Keywords: anti-NMDA receptor encephalitis, autoimmune encephalitis, rehabilitation, cognition deficits

\section{Introduction}

Anti- $N$-methyl-D-aspartate (anti-NMDA) receptor encephalitis is an acute, immune-mediated paraneoplastic syndrome with a characteristic sequential clinical course. It includes a prodrome of flu-like illness progressing to behavior changes, psychosis, and memory deficit. Dyskinesia, seizure attacks, deteriorated consciousness level, and autonomic instability may be seen in later stages. ${ }^{1-4}$ Patients may gradually recover, with these symptoms resolving in reverse order. ${ }^{1-4}$ Dalmau's group first described anti-NMDA receptor encephalitis in 2005 and suggested that it was an acute, female-predominant (81\%) autoimmune encephalitis. ${ }^{1,2}$ About half of the patients with anti-NMDA receptor encephalitis had underlying neoplasms, with ovarian teratomas (94\%) being the most common. ${ }^{1-3}$ A recent large cohort study demonstrated that milder severity of symptoms, no need for intensive care, early immunotherapy, and prompt tumor removal were good prognostic factors. ${ }^{3}$ Half of the patients had improvement in symptoms within 4 weeks after early immunotherapy. In another case report, the authors noted that steroid usage 3 months after onset of symptoms also resulted in significant improvements. ${ }^{3,5}$ The recovery phase might last for 18 months or longer. ${ }^{3-4}$ To maximize a patient's functional outcome, clinical practitioners need to be aware of this potentially curable disease and its common sequelae in order to organize rational, individualized rehabilitation programs. ${ }^{6,7}$ We report the long-term course of a young pediatric patient with recalcitrant anti-NMDA receptor encephalitis
Correspondence: Yu-Ching Lin Department of Physical Medicine and Rehabilitation, National Cheng Kung University Hospital, College of Medicine, National Cheng Kung University,

I38 Sheng-Li Road, Tainan 704, Taiwan

Tel +88662353535 Ext 2666

Fax +88662766106

Email richelin@mail.ncku.edu.tw
Neuropsychiatric Disease and Treatment 2014:10 2263-2267 
and prolonged length of stay in hospital for 7 months. She eventually retained independent functional performance after extensive immunotherapy and rehabilitation.

\section{Case report}

A young girl aged 3 years 2 months and without known medical or psychiatric history was admitted to our hospital due to personality changes that had persisted for 2 weeks. Her condition progressed over the course of 12 days to behavioral regression, unsteady gait, headache, irritable crying, catatonia-like response, generalized choreoathetoid movements, orofacial dyskinesia, and collapsed lung, in successive order. As a result, she was transferred to the pediatric intensive care unit. Cerebrospinal fluid analysis revealed pleocytosis with predominance of lymphocytes. Her brain magnetic resonance imaging (MRI) found leptomeningeal enhancement at bilateral frontoparietal area, nodular enhancement along the tentorium, and some tiny white-matter lesions over bilateral frontal lobes (Figure 1). Global cortical dysfunction without epileptiform discharge was seen on the electroencephalogram. A complete etiologic investigation showed insufficient evidence of bacterial

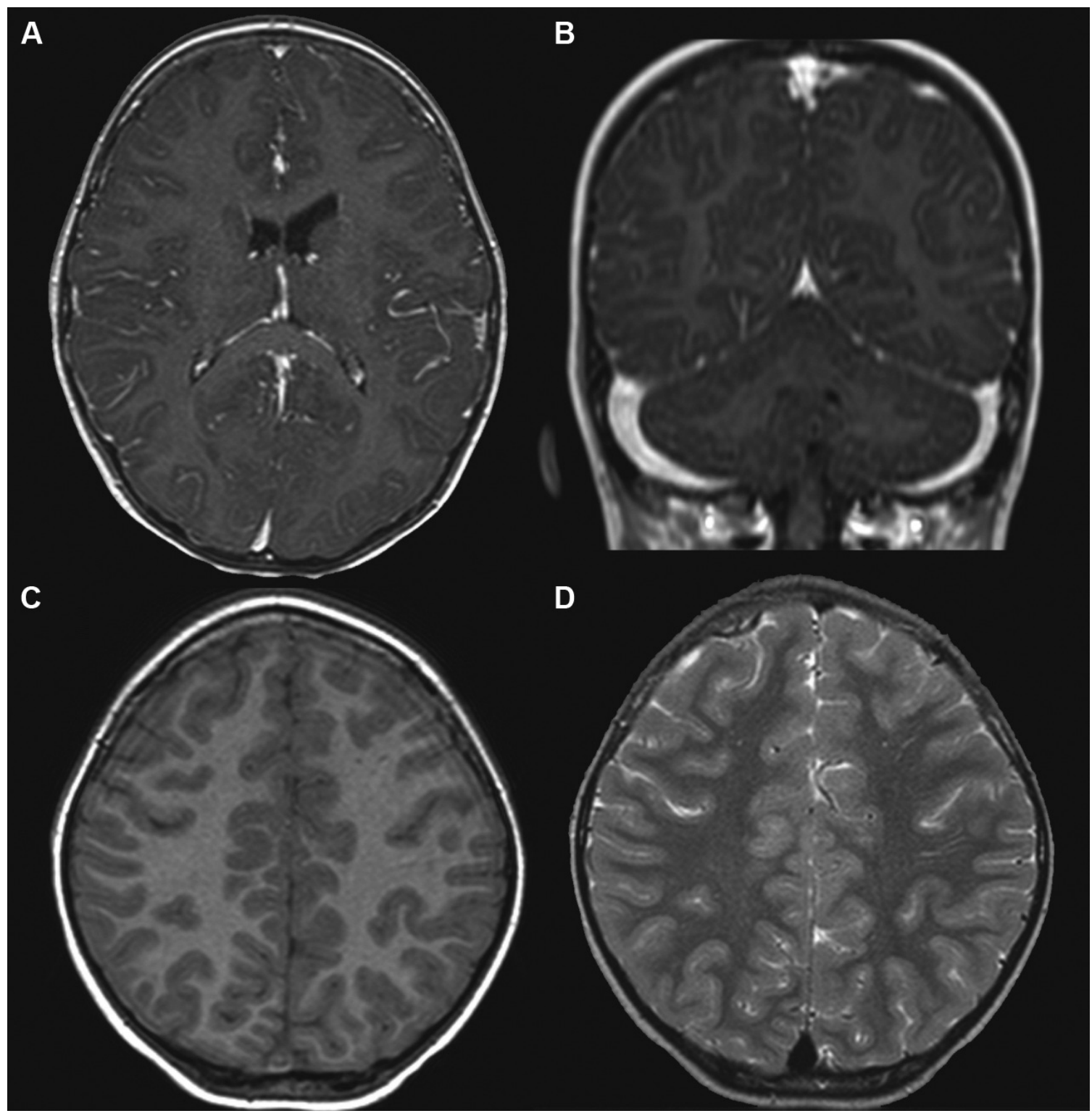

Figure I Brain MRI of a 3-year-old girl with anti- $N$-methyl-D-aspartate receptor encephalitis.

Notes: TI-weighted with gadolinium-enhanced MRI revealed (A) prominence of leptomeningeal enhancement at bilateral frontoparietal areas (axial view) and (B) nodular enhancement along the tentorium edge (coronal view). (C) TI-weighted (axial view) and (D) T2-weighted (axial view) MRI showing tiny white-matter lesions over bilateral frontal lobes.

Abbreviation: MRI, magnetic resonance imaging. 
infection, electrolyte imbalance, intoxication, or endocrine disturbances.

At first, under the clinical suspicion of meningoencephalitis, she was empirically treated with acyclovir plus azithromycin. After a cerebrospinal fluid NMDA receptor antibody study reported positive results, the antibiotics were shifted to intravenous immunoglobulin for two courses, followed by pulse methylprednisolone therapy $(30 \mathrm{mg} / \mathrm{kg} / \mathrm{dose}$, four doses) and maintenance oral prednisolone $(0.9 \mathrm{mg} / \mathrm{kg} /$ day $)$. Extensive neoplasm surveys, including abdominal computed tomography scan, were all unremarkable. Aripiprazole and midazolam sedation were added for control of additional symptoms. Her choreoathetosis and orofacial dyskinesia recovered partially for several weeks, but these symptoms reappeared after we discontinued prednisolone because of her episodic sepsis, autonomic instability, and poorlycontrolled gastrointestinal bleeding. The pediatric neurologist prescribed plasmaphoresis about 3 months after onset of symptoms, but it was discontinued because of anaphylactic reactions and bilateral pneumonia with collapsed lung. She received a combination therapy of weekly rituximab (375 mg/m $\mathrm{m}^{2}$, four doses) and prolonged monthly cyclophosphamide pulse therapy ( $750 \mathrm{mg} / \mathrm{m}^{2}, 8$ months), followed by azathioprine for 3 months.

For her inpatient rehabilitation program, passive rangeof-motion exercise was performed for her during periods of protracted sedation to prevent contracture. After cyclophosphamide and rituximab was applied for 6 weeks, her troublesome generalized choreoathetosis attenuated gradually. We started intensive facilitation and postural training after tapering off the sedative agents. In order to prevent oral dyskinesia-related aspiration and pneumonia, we performed temporary percutaneous endoscopic gastrostomy.

The patient was discharged after a 7-month period of hospitalization, which required maximal assistance in mobility, self-care, and cognition domains as measured with the Functional Independence Measure for Children. For her outpatient rehabilitation, we arranged fixed physical, occupational, and speech therapists with whom she was familiar, and we placed her in an isolated setting to avoid distracting stimuli that might deteriorate her attention span, emotional status, and behavioral outbursts. At 12 months after the onset of symptoms, she had merely achieved the developmental age of 6-8 months in terms of gross-motor and fine-motor milestones and 9-12 months in terms of cognition. She could obey some simple verbal orders but was mute most of the time, with cognitive deficits considered to be the main cause. Over the next 6 months, sequential programs such as toy-based training for postural control, imitation learning, matching training, and treadmill ambulation with partial body-weight support produced significant improvement. Her gross-motor skills progressed to the developmental milestone of 24-27 months, her fine-motor movements improved to 20-23 months, and her cognition advanced to 24-29 months. She could deal with simple self-care tasks, but occasional incontinence was still noted. She could speak more than five words and expressed herself correspondingly. The physical therapy with tricycle riding and balance-beam exercise continued according to the patient's tolerance.

In order to improve her cognitive deficits, psychological evaluation and training such as cognitive-behavioral therapy for attention, memory, and emotion regulation were added to her programs. The initial assessment for her intelligence quotient (IQ), measured by Wechsler Preschool and Primary Scale of Intelligence, was reported as mild mental retardation (full IQ =62 ), with performance IQ out of proportion to her scale in verbal IQ (scores were 58 and 71, respectively).

After 28 months of rehabilitation, the patient reached full development in gross-motor milestones, regained control of continence sphincters, and was able to communicate in complete sentences with improved reasoning. Three years after onset of anti-NMDA receptor encephalitis, the IQ test showed no significant change except for performance IQ improvement from 58 to 64 . She still had residual sequelae of mild mental retardation, and she had a mildly impulsive personality and was easily distracted.

\section{Discussion}

The typical presentations of anti-NMDA receptor encephalitis could be divided into eight categories in decreasing order of prevalence, including acute behavioral or cognitive change, movement disorder, memory deficit, speech disorder, seizure, decreased level of consciousness, autonomic dysfunction, and central hypoventilation. In an observational cohort study, about $87 \%$ of patients shared similar symptoms that included four or more of the eight categories within the first 4 weeks. ${ }^{3}$ Definite diagnosis for this disease was the presence of NMDA receptor antibodies in the patient's cerebrospinal fluid (100\% sensitivity) or serum ( $85 \%$ sensitivity). ${ }^{2,3}$ Reduced levels of cerebrospinal fluid antibodies appeared to be correlated to a better clinical outcome. ${ }^{2,8}$ There were no correlations between different domains of symptoms, extent of disease progression, and brain MRI findings, with only $33 \%-50 \%$ of patients showing nonspecific abnormalities on standard MRI. ${ }^{2,3}$ One recent 
functional MRI study proposed that reduced functional connectivity between bilateral hippocampus may predict memory performance, and that extensive white-matter changes in diffusion tensor imaging might correlate to the disease severity. ${ }^{9}$ The acronym "NMDAR" is useful as a mnemonic for remembering the symptoms of anti-NMDA receptor encephalitis: "N" for nonspecific prodrome, "M" for memory and cognition impairment, " $D$ " for dystonic posture, "A" for autonomic dysfunction, and "R" for respiration depression. Early detection of this disease and confirmed diagnosis with NMDA receptor antibodies ensures early intervention and better recovery.

The recommended first-line immunotherapies were corticosteroid, intravenous immunoglobulin, and plasmaphoresis. About $60 \%$ of patients achieved good outcomes with minor functional deficits remaining after first-line therapy or tumor removal. ${ }^{3}$ The second-line immunotherapies, which include rituximab and cyclophosphamide, were recommended to be started if patients failed to respond to first-line therapies after no more than 4 weeks, ${ }^{2,3}$ and resulted in a response rate of approximately $78 \%{ }^{3}$ Full recovery and fewer neurological relapses were more frequently seen in patients with teratoma that had been removed. ${ }^{2,3,10}$ The general relapsing rate was $12 \%-24 \%$ within the first 2 years, and overall mortality rate was $4 \%-9.5 \% .^{2,3}$

A search of relevant articles was conducted on PubMed (from 2000 to August 2014), Medline (from 2000 to August 2014) and Embase (from 2000 to August 2014), using "Anti-NMDA receptor encephalitis" or "autoimmune encephalitis" as search terms. In the current literature, the age of onset for anti-NMDA receptor encephalitis ranged from 8 months to 85 years. ${ }^{3,11}$ Adult patients more often presented with memory deficits, autonomic dysfunction, and central hypoventilation. In contrast, children younger than 12 years of age generally presented with behavioral or cognitive changes, involuntary movements, speech disorders, ataxia, and hemiparesis. ${ }^{3}$ Consistent with recent literature for young patients with anti-NMDA receptor encephalitis, we did not identify any underlying tumor for our patient. Her unique features were prolonged intractable oral dyskinesia and generalized dystonia for 6 months. These symptoms even persisted an additional 6 weeks after the start of second-line immunotherapy. The severity and duration of the movement disorders might be a reflection of the encephalitis severity. ${ }^{3,6}$ It could be worthwhile to arrange a comprehensive treatment and rehabilitation program for ill children who do not fit the good-outcome predictors, because their prognosis might still be favorable, such as the case we have reported.
The ideal treatment goals for patients with anti-NMDA receptor encephalitis are to accomplish stable medical condition, attain functional goals, and achieve good family training. ${ }^{6}$ The children in several studies had multiple impairments at the time of hospital discharge, especially deficits in delicate motor skills and cognition, and were deemed as needing further outpatient rehabilitation. ${ }^{6,7,12}$ Goal-oriented training based on a multidisciplinary approach and individualized programs in physical, occupational, psychological, and speech training provided better functional outcomes and might shorten the rehabilitation time course. ${ }^{6,7}$ In the current case, physical performance, nonverbal reasoning, and fluid intelligence were often impaired but all of them had fair to good response to training. On the contrary, problems such as the patient's short attention span were frustrating. Because cognitive recovery was significantly impaired despite recovery in other neurological deficits, compensating cognitive deficits might be a core concept for late-phase rehabilitation. ${ }^{6}$ Family education about rational goals and the importance of strictly carrying out cognitive-behavioral therapy at home might also be helpful. Moreover, an individualized education program to address a child's distraction, delayed information interpretation speed, and learning difficulty is also suggested to be executed in school. ${ }^{6}$

Most articles about anti-NMDA receptor encephalitis in the literature described the presentation of symptoms, diagnostic process, follow-up antibody titers, and neurological status. However, none of them described long-term rehabilitation strategies for these patients; only two articles described inpatient rehabilitation programs in seven patients. ${ }^{6,7}$ Our report highlights the sequential functional changes experienced by the young patient during the 28 months of rehabilitation (both inpatient and outpatient rehabilitation), and pointed out the possible challenges during the rehabilitative programs. Even so, the interventions and therapies described here may not be useful for all patients with anti-NMDA receptor encephalitis.

In cases of encephalitis of unknown cause and with characteristic symptoms, treatable anti-NMDA receptor encephalitis should be taken into consideration and further intervention must be performed as early as possible. Comprehensive multidisciplinary rehabilitation programs, including physical, occupational, psychological, and speech training, are usually necessary for these patients and, generally, a good outcome can be expected.

\section{Acknowledgment}

We thank Dr Arthur Chou for his assistance in revision of English. 


\section{Disclosure}

The authors received no financial support for the research, authorship, and/or publication of this article.

No previous presentations of this research have been made, either in manuscript or abstract form. All authors have read the final manuscript draft and approve it for submission.

The authors have declared no potential conflicts of interest with respect to the research, authorship, and/or publication of this article. The authors report no other conflicts of interest in this work.

\section{References}

1. Vitaliani R, Mason W, Ances B, Zwerdling T, Jiang Z, Dalmau J. Paraneoplastic encephalitis, psychiatric symptoms, and hypoventilation in ovarian teratoma. Ann Neurol. 2005;58(4):594-604.

2. Dalmau J, Gleichman AJ, Hughes EG, et al. Anti-NMDA-receptor encephalitis: case series and analysis of the effects of antibodies. Lancet Neurol. 2008;7(12):1091-1098.

3. Titulaer MJ, McCracken L, Gabilondo I, et al. Treatment and prognostic factors for long-term outcome in patients with anti-NMDA receptor encephalitis: an observational cohort study. Lancet Neurol. 2013; 12(2): 157-165

4. Iizuka T, Sakai F, Ide T, et al. Anti-NMDA receptor encephalitis in Japan: long-term outcome without tumor removal. Neurology. 2008;70(7): 504-511.
5. Turkdoğan D, Orengul AC, Zaimoğlu S, Ekinci G. Anti- $N$-methylD-aspartate (Anti-NMDA) receptor encephalitis: rapid and sustained clinical improvement with steroid therapy starting in the late phase. J Child Neurol. 2014;29(5):684-687.

6. Houtrow AJ, Bhandal M, Pratini NR, Davidson L, Neufeld JA. The rehabilitation of children with anti- $N$-methyl-D-aspartate-receptor encephalitis: a case series. Am J Phys Med Rehabil. 2012;91(5):435-441.

7. Tham SL, Kong KH. A case of anti-NMDAR ( $N$-methyl-D-aspartate receptor) encephalitis: a rehabilitation perspective. Neuro Rehabilitation. 2012;30(2):109-112.

8. Frechette ES, Zhou L, Galetta SL, Chen L, Dalmau J. Prolonged follow-up and CSF antibody titers in a patient with anti-NMDA receptor encephalitis. Neurology. 2011;76(7 Suppl 2):S64-S66.

9. Finke C, Kopp UA, Scheel M, et al. Functional and structural brain changes in anti- $N$-methyl-D-aspartate receptor encephalitis. Ann Neurol. 2013;74(2):284-296.

10. Florance NR, Davis RL, Lam C, et al. Anti- $N$-methyl-D-aspartate receptor (NMDAR) encephalitis in children and adolescents. Ann Neurol. 2009;66(1):11-18.

11. Cantarín-Extremera V, Duat-Rodríguez A, González-GutiérrezSolana L, López-Marín L, Armangue T. Clinical case of anti$\mathrm{N}$-methyl-D-aspartate receptor encephalitis in an 8-month-old patient with hyperkinetic movement disorder. Pediatr Neurol. 2013;48(5):400-402.

12. Biancheri R, Pessagno A, Baglietto MG, et al. Anti- $N$-methyl-Daspartate-receptor encephalitis in a four-year-old girl. $J$ Pediatr 2010;156(2):332-334
Neuropsychiatric Disease and Treatment

\section{Publish your work in this journal}

Neuropsychiatric Disease and Treatment is an international, peerreviewed journal of clinical therapeutics and pharmacology focusing on concise rapid reporting of clinical or pre-clinical studies on a range of neuropsychiatric and neurological disorders. This journal is indexed on PubMed Central, the 'PsycINFO' database and CAS,

\section{Dovepress}

and is the official journal of The International Neuropsychiatric Association (INA). The manuscript management system is completely online and includes a very quick and fair peer-review system, which is all easy to use. Visit http://www.dovepress.com/testimonials.php to read real quotes from published authors. 